\title{
Speed-Sensorless Vector Torque Control of Induction Machines Using a Two-Time-Scale Approach
}

\author{
Heath Hofmann, Student Member, IEEE, and Seth R. Sanders, Member, IEEE
}

\begin{abstract}
Conditions for observability of a linearized smoothairgap induction machine model are examined, and limitations of any speed-sensorless observer scheme based on such a model are discussed. An approach for speed-sensorless flux estimation based on singular perturbation theory is developed. This approach relies on the natural time-scale separation between the electrical and mechanical dynamics of the induction machine. A fullorder observer of an induction machine is presented, incorporating a correction term which has an intuitive explanation when one considers steady-state stator currents. Using singular perturbation theory, convergence of the observer is shown for all open-loop stable operating points of the induction machine, with the exception of dc excitation. Sensitivity of the observer to parameter deviations is discussed. Experimental results are presented confirming the validity of the above approach.
\end{abstract}

Index Terms - Induction machines, singular perturbation theory, speed-sensorless torque control.

\section{INTRODUCTION}

$\mathbf{M}$ ANY implementations of speed-sensorless flux estimation schemes are based on the so-called back-EMF approach, which assumes a smooth-airgap model of an induction machine and requires measurements of only stator voltage and/or current. Several papers [1]-[5] present experimental data showing the effectiveness of these methods over a wide range of operating conditions. However, these articles do not consider the effect of operating point on convergence of the estimator in their stability analyses. For example, none of these methods can converge for dc excitation.

We propose an approach to speed-sensorless flux estimation using singular perturbation theory [6], which is based on the presumption that the electrical variables of an induction machine have significantly faster dynamics than the mechanical variables. As such, the flux and current dynamics evolve on a time scale on which the rotor speed appears constant. Consistently, the mechanical rotor dynamics can be analyzed under the assumption that the electrical dynamics are converged to a quasi-steady-state value, which is a function of the slowly varying rotor speed. These assumptions allow us to study the electrical and mechanical dynamics separately,

Paper IPCSD 97-63, presented at the 1996 Industry Applications Society Annual Meeting, San Diego, CA, and approved for publication in the IEEE TRANSACTIONS ON INDUSTRY APPLICATIONS by the Industrial Drives Committee of the IEEE Industry Applications Society. This work was supported by the National Science Foundation under Grant ECS-9358284. Manuscript released for publication September 12, 1997.

The authors are with the Department of Electrical Engineering and Computer Science, University of California, Berkeley, CA 94720-1772 USA (e-mail: heath@eecs.berkeley.edu; sanders@eecs.berkeley.edu).

Publisher Item Identifier S 0093-9994(98)01204-3. simplifying analysis. This approach for analyzing induction machine dynamics is discussed in [7]. We believe that singular perturbation theory is a natural approach to studying electrical machine systems.

We begin with a brief introduction to singular perturbation theory, which provides a framework for studying two-timescale systems. Next, we discuss the conditions necessary for small-signal observability of an induction machine model using linear system theory. This allows conclusions on the limitations of any estimator based on the smooth-airgap model. We then develop a full-order observer of an induction machine. The observer incorporates a correction term which is injected into the observer subsystem corresponding to the mechanical dynamics. We use singular perturbation theory to show that the observer converges asymptotically for all stable steady-state operating points, with the exception of dc excitation, provided the mechanical dynamics are slow compared to the electrical dynamics. We then analyze sensitivity of the observer to parameter deviations. Experimental data verifies the technique.

\section{Singular Perturbation Theory}

The two-time-scale approach can be applied to systems where the state variables can be split into two sets, one having "fast" dynamics, the other having "slow" dynamics. The difference between the two sets of dynamics can be distinguished by the use of a small multiplying scalar $\epsilon$. A linear state-space system with appropriate structure is represented by

$$
\begin{aligned}
\frac{d}{d t} x_{f} & =\mathbf{A}_{11} x_{f}+\mathbf{A}_{12} x_{s} \\
\frac{d}{d t} x_{s} & =\epsilon\left(\mathbf{A}_{21} x_{f}+\mathbf{A}_{22} x_{s}\right) .
\end{aligned}
$$

The small parameter $\epsilon$ is introduced to emphasize that the portion of the state vector represented by $x_{s}$ evolves on a slower time scale than that represented by $x_{f}$. In the limiting case, as $\epsilon \rightarrow 0$, the slow variables $x_{s}$ become constant. Provided the fast dynamics are stable (eigenvalues of $\mathbf{A}_{11}$ have negative real parts), the fast variables converge to a quasisteady-state value that is dependent upon the slow variables, i.e.,

$$
x_{f}=-\mathbf{A}_{11}^{-1} \mathbf{A}_{12} x_{s} .
$$

When studying the slow dynamics, we shift time frames by introducing a new time scale $\tau=\epsilon t$. The dynamics then 
become

$$
\begin{aligned}
\frac{d}{d \tau} x_{f} & =\frac{1}{\epsilon}\left(\mathbf{A}_{11} x_{f}+\mathbf{A}_{12} x_{s}\right) \\
\frac{d}{d \tau} x_{s} & =\mathbf{A}_{21} x_{f}+\mathbf{A}_{22} x_{s}
\end{aligned}
$$

Then, in the limiting case, where $\epsilon \rightarrow 0$, the fast dynamics become so fast that $x_{f}$ can be considered to be instantaneously determined by (3). The slow dynamics can then be written as

$$
\frac{d}{d \tau} x_{s}=\left(-\mathbf{A}_{21} \mathbf{A}_{11}^{-1} \mathbf{A}_{12}+\mathbf{A}_{22}\right) x_{s}=\mathbf{A}_{s} x_{s} .
$$

Singular perturbation theory provides a framework for this simplifying analysis. Specifically provided $\epsilon$ is small enough, conclusions drawn from the separate analyses of reduced subsystems concerning stability, observability, etc., also apply to the underlying system [6]. In the context of our analysis of induction machine dynamics, we consider the electromagnetic variables as "fast" and the mechanical variables as "slow."

\section{INDUCTION MACHINE MODEL}

The notation used throughout the remainder of this paper is presented in Table I. We use a standard two-axis smoothairgap model for the induction machine, with stator current and rotor flux as the electrical state variables and rotor speed and load torque as the mechanical state variables:

$$
\begin{aligned}
\frac{d}{d t}\left[\begin{array}{l}
\imath_{s} \\
\lambda_{r}
\end{array}\right] & =\mathbf{A}_{f}\left(\dot{\rho}, \omega_{r}\right)\left[\begin{array}{c}
v_{s} \\
\lambda_{r}
\end{array}\right]+\left[\begin{array}{c}
\frac{L_{r}}{\sigma^{2}} v_{s} \\
\mathbf{0}
\end{array}\right] \\
\frac{d}{d t} \omega_{r} & =\frac{1}{H}\left(\tau_{e}-\tau_{\ell}-B \omega_{r}\right) \\
\frac{d}{d t} \tau_{\ell} & =0 \\
\tau_{e} & =\frac{M}{L_{r}} \imath_{s}^{T} \mathbf{J} \lambda_{r}
\end{aligned}
$$

$$
\begin{aligned}
& \mathbf{A}_{f}\left(\dot{\rho}, \omega_{r}\right)= \\
& \left.\qquad \begin{array}{cc}
-\frac{\left(R_{s} L_{r}^{2}+R_{r} M^{2}\right)}{\sigma^{2} L_{r}} \mathbf{I}-\dot{\rho} \mathbf{J} & \frac{M}{\sigma^{2}}\left(\frac{R_{r}}{L_{r}} \mathbf{I}-\omega_{r} \mathbf{J}\right) \\
\frac{R_{r} M}{L_{r}} \mathbf{I} & -\frac{R_{r}}{L_{r}} \mathbf{I}-\left(\dot{\rho}-\omega_{r}\right) \mathbf{J}
\end{array}\right]
\end{aligned}
$$

$$
\mathbf{I}=\left[\begin{array}{ll}
1 & 0 \\
0 & 1
\end{array}\right] ; \quad \mathbf{J}=\left[\begin{array}{cc}
0 & -1 \\
1 & 0
\end{array}\right]
$$

The variable $\rho$ represents the angle corresponding to the chosen reference frame of the system. In the stationary reference frame, $\dot{\rho}=0$, and in the electrical reference frame $\dot{\rho}=\omega_{e}$. We denote variables in the electrical reference frame with a superscript $e$ (e.g., $\left.\imath_{s}^{e}\right)$. For the following analysis, we choose the stator voltage as the reference vector in the electrical reference frame, hence, $v_{s d}^{e}=\left\|v_{s}\right\|$ and $v_{s q}^{e}=0$.

We denote steady-state electrical variables with a tilde (e.g., $\tilde{\imath}_{s}^{e}$ ). In electrical steady state, we can write the stator
TABLE I

Notation-Parameters Given Were Taken From a Four-Pole Induction Machine Rated at $3 \mathrm{HP}, 60 \mathrm{~Hz}, 220$ V Line-Line, AND 9A

$$
\begin{aligned}
& \text { Electrical } \\
& \lambda_{r}=\left[\begin{array}{ll}
\lambda_{r d} & \lambda_{r q}
\end{array}\right]^{T} \\
& \imath_{s}=\left[\begin{array}{ll}
l_{s d} & \imath_{s q}
\end{array}\right]^{T} \\
& v_{s}=\left[\begin{array}{ll}
v_{s d} & v_{s q}
\end{array}\right]^{T} \\
& \omega_{e}
\end{aligned}
$$

Electrical Parameters $R_{s}=1.59 \Omega$

$R_{\tau}=1.86 \Omega$

$L_{s}=116.5 \mathrm{mH}$

$L_{r}=116.7 m H$

$M=109.5 m H$

$\sigma^{2}=L_{s} L_{r}-M^{2}$

$L_{\ell s}=L_{s}-M$

$L_{\ell r}=L_{r}-M$

Mechanical Variables

$\omega_{T}$

$\omega_{s}=\omega_{e}-\omega_{r}$

$\tau_{e}=\frac{M}{L_{r}} \imath_{s}^{T} \mathbf{J} \lambda_{r}$

$\tau_{\ell}$

Mechanical Parameters

$H=0.8 \mathrm{~kg} \mathrm{~m} \mathrm{~m}^{2}$

$B=0.1 \frac{\mathrm{kg} \mathrm{m}}{\mathrm{s}}$

Matrix Notation

I

$\mathbf{J}$

$\mathbf{0}_{m \times n}$

\section{Rotor Flux Vector \\ Stator Current Vector \\ Stator Voltage Vector \\ Electrical Frequency}

Stator Resistance

Rotor Resistance

Stator Inductance

Rotor Inductance

Mutual Inductance

Leakage Term

Stator Leakage Inductance

Rotor Leakage Inductance

\section{Rotor Velocity \\ Slip Frequency \\ Electromagnetic Torque \\ Load Torque}

\section{Moment of Inertia of Rotor \\ Damping Constant of Rotor}

$2 \times 2$ Identity Matrix

Orthogonal Rotation Matrix

Matrix of zeroes with $m$ rows

and $n$ columns current and rotor flux as a function of stator voltage, electrical frequency, and rotor speed:

$$
\left[\begin{array}{c}
\tilde{\nu}_{s}^{e} \\
\tilde{\lambda}_{r}^{e}
\end{array}\right]=-\mathbf{A}_{f}^{-1}\left(\omega_{e}, \omega_{r}\right)\left[\begin{array}{c}
\frac{L_{r}}{\sigma^{2}} v_{s}^{e} \\
0
\end{array}\right] .
$$

Note that we include mechanical dynamics in our model, where the machine load is modeled by a constant load torque term and a linear damping term. Although the assumption of constant load torque is made here and in the analysis of the observer, the experimental data show that the observer is also effective with other loads.

\section{OBSERVABILITY OF IndUCtion Machine}

We now analyze the conditions under which the model of the smooth-airgap induction machine is observable. First, we note that the model is unobservable if the rotor flux is identically zero, because the rotor speed is introduced into the electrical dynamics through the back-EMF $\lambda_{r}^{e} \omega_{r}$. However, in steady state, the rotor flux is given by

$$
\begin{aligned}
\tilde{\lambda}_{r}^{e}= & \frac{R_{r} M}{\left(R_{s} \omega_{s} L_{r}+R_{r} \omega_{e} L_{s}\right)^{2}+\left(R_{s} R_{r}-\sigma^{2} \omega_{e} \omega_{s}\right)^{2}} \\
& \times\left[\left(R_{r} R_{s}-\omega_{e} \omega_{s} \sigma^{2}\right) \mathbf{I}-\left(\omega_{e} R_{r} L_{s}+\omega_{s} R_{s} L_{r}\right) \mathbf{J}\right] v_{s}^{e}
\end{aligned}
$$

hence, the steady-state rotor flux is zero only in the trivial case of zero stator voltage excitation and, so, in the following analysis, we assume $\tilde{\lambda}_{r}^{e} \neq \mathbf{0}$. 
We study small-signal observability by linearizing the above induction machine model about an operating point and applying the concepts of observability from linear system theory [8]. We study the system in the electrical reference frame, hence, the resulting linearized model will be time invariant, although dependent upon operating point. The linearized induction machine dynamics are given by

$$
\begin{aligned}
\frac{d}{d t} \Delta x^{e} & =\mathbf{A}_{\ell}\left(\tilde{\mathbf{x}}^{e}\right) \Delta x^{e}+\frac{L_{r}}{\sigma^{2}}\left[\begin{array}{c}
\Delta v_{s}^{e} \\
\mathbf{0}
\end{array}\right] \\
\Delta v_{s}^{e} & =\mathbf{C}_{\ell} \Delta x^{e}
\end{aligned}
$$

$$
\begin{aligned}
& \mathbf{A}_{\ell}\left(x^{e}\right)= \\
& {\left[\begin{array}{c|c|c|c}
\mathbf{A}_{f}\left(\omega_{e}, \omega_{r}\right) & \mathbf{0}_{2 \times 1} & \mathbf{0}_{2 \times 1} \\
\hline \mathbf{J} \lambda_{r}^{e} & \mathbf{0}_{2 \times 1} \\
\hline-\frac{M}{H L_{r}} \lambda_{r}^{e T} \mathbf{J} & \frac{M}{H L_{r}} \imath_{s}^{e T} \mathbf{J} & -\frac{B}{H} & -\frac{1}{H} \\
\hline \mathbf{0}_{1 \times 2} & \mathbf{0}_{1 \times 2} & 0 & 0
\end{array}\right]}
\end{aligned}
$$

$$
\begin{gathered}
x^{e}=\left[\begin{array}{c}
v_{s}^{e} \\
\lambda_{r}^{e} \\
\omega_{r} \\
\tau_{l}
\end{array}\right] \\
\mathbf{C}_{\ell}=\left[\begin{array}{ll}
\mathbf{I} & \mathbf{0}_{2 \times 4}
\end{array}\right] .
\end{gathered}
$$

To test for observability we form the observability matrix:

$$
\mathcal{C}_{o}=\left[\begin{array}{c}
\mathbf{C}_{\ell} \\
\mathbf{C}_{\ell} \mathbf{A}_{\ell} \\
\mathbf{C}_{\ell} \mathbf{A}_{\ell}^{2} \\
\mathbf{C}_{\ell} \mathbf{A}_{\ell}^{3} \\
\mathbf{C}_{\ell} \mathbf{A}_{\ell}^{4} \\
\mathbf{C}_{\ell} \mathbf{A}_{\ell}^{5}
\end{array}\right] .
$$

The observability matrix $\mathcal{C}_{o}$ for the linearized induction machine model in variable form is too large to be included in this paper (in our analysis, the matrix was generated using the analytical software MAPLE).

The linearized system is observable if and only if there is no vector $a \neq \mathbf{0}$ that satisfies

$$
\mathcal{C}_{o} a=\mathbf{0}_{12 \times 1} \text {. }
$$

As shown in the Appendix, the vector

$$
a=\left[\begin{array}{c}
\mathbf{0}_{2 \times 1} \\
\left(\frac{R_{r}}{L_{r}} \mathbf{I}-\omega_{r} \mathbf{J}\right)^{-1} \mathbf{J} \lambda_{r}^{e} \\
1 \\
-B-\frac{M}{R_{r}^{2}+\omega_{r}^{2} L_{r}^{2}}\left[{ }_{s}^{e T}\left(R_{r} \mathbf{I}+\omega_{r} L_{r} \mathbf{J}\right) \lambda_{r}^{e}\right]
\end{array}\right]
$$

is the only vector that can satisfy (21) and does so only if the electrical frequency $\omega_{e}$ is zero. Hence, the small-signal model of the smooth-airgap induction machine model is observable for all operating points, except for dc excitation. In the case of dc excitation, $\imath_{s}=v_{s} / R_{s}$ and, hence, no information about the rotor dynamics (i.e., speed and/or torque) is available. We note that observability of the small-signal model is the requirement for arbitrary assignment of the observer eigenvalues.

\section{Two-Time-Scale Approach to ObSERVER Design}

We denote observer variables with a hat (e.g., $\hat{\imath}_{s}$ ). The full-order observer is given by

$$
\frac{d}{d t}\left[\begin{array}{l}
\hat{\imath}_{s} \\
\hat{\lambda}_{r}
\end{array}\right]=\mathbf{A}_{f}\left(\dot{\rho}, \hat{\omega}_{r}\right)\left[\begin{array}{l}
\hat{\imath}_{s} \\
\hat{\lambda}_{r}
\end{array}\right]+\left[\begin{array}{l}
\frac{L_{r}}{\sigma^{2}} v_{s} \\
\mathbf{0}_{2 \times 1}
\end{array}\right]
$$

$$
\begin{aligned}
\frac{d}{d t} \hat{\omega}_{r} & =\frac{1}{H}\left\{\hat{\tau}_{e}-\hat{\tau}_{\ell}-B \hat{\omega}_{r}-K_{\omega} f_{c x}\left(\imath_{s}, \hat{\imath}_{s}, v_{s}^{e}, \omega_{e}\right)\right\} \\
\frac{d}{d t} \hat{\tau}_{\ell} & =\frac{1}{H} K_{\tau} f_{c x}\left(\imath_{s}, \hat{\imath}_{s}, v_{s}^{e}, \omega_{e}\right) \\
\hat{\tau}_{e} & =\frac{M}{L_{r}} \hat{\imath}_{s}^{T} \mathbf{J} \hat{\lambda}_{r}
\end{aligned}
$$

where $f_{c x}\left(\imath_{s}, \hat{\imath}_{s}, v_{s}^{e}, \omega_{e}\right)$ is an injection term designed to correct the mechanical dynamics of the observer and will be discussed in more detail in the following.

We denote the error between observer and machine variables using the convention $\delta x=\hat{x}-x$. The nonlinear error dynamics are then given by

$$
\frac{d}{d t}\left[\begin{array}{l}
\delta r_{s} \\
\delta \lambda_{r}
\end{array}\right]=\mathbf{A}_{f}\left(\dot{\rho}, \omega_{r}\right)\left[\begin{array}{l}
\delta u_{s} \\
\delta \lambda_{r}
\end{array}\right]+\left[\begin{array}{c}
-\frac{M}{\sigma^{2}} \mathbf{J} \hat{\lambda}_{r} \\
\mathbf{J} \hat{\lambda}_{r}
\end{array}\right] \delta \omega_{r}
$$

$$
\begin{aligned}
\frac{d\left(\delta \omega_{r}\right)}{d t} & =\frac{1}{H}\left\{\delta \tau_{e}-\delta \tau_{\ell}-B \delta \omega_{r}-K_{\omega} f_{c x}\left(\imath_{s}, \hat{\imath}_{s}, v_{s}^{e}, \omega_{e}\right)\right\} \\
\frac{d\left(\delta \tau_{\ell}\right)}{d t} & =\frac{1}{H} K_{\tau} f_{c x}\left(\imath_{s}, \hat{\imath}_{s}, v_{s}^{e}, \omega_{e}\right) .
\end{aligned}
$$

We now consider convergence of the observer. The following analysis is limited to small-signal error dynamics of the observer about a steady-state operating point. Although small-signal stability is a minimal requirement, experiments characterizing the response of the observer show that it also works under large transient conditions.

We prove convergence of the observer using singular perturbation theory [6]. With this approach, we assume that the electrical error dynamics (27) are significantly faster than the mechanical error dynamics (28) and (29). Hence, when analyzing the electrical error dynamics, we thus assume that the rotor speed error is essentially constant. Analysis presented in [9] reveals that, for any electrical frequency $\omega_{e}$ and rotor speed $\omega_{r}$, the matrix $\mathbf{A}_{f}\left(\omega_{e}, \omega_{r}\right)$ is exponentially stable. Hence, the electrical error dynamics converge to a quasisteady-state value which is a function of the rotor speed error:

$$
\left[\begin{array}{l}
\delta \tilde{u}_{s}^{e} \\
\delta \tilde{\lambda}_{r}^{e}
\end{array}\right]=-\mathbf{A}_{f}^{-1}\left(\omega_{e}, \omega_{r}\right)\left[\begin{array}{c}
-\frac{M}{\sigma^{2}} \mathbf{J} \\
\mathbf{J}
\end{array}\right] \tilde{\lambda}_{r}^{e} \delta \omega_{r}+O\left(\delta \omega_{r}^{2}\right) .
$$

For this reason, no injection term is used in the electrical subsystem dynamics. 


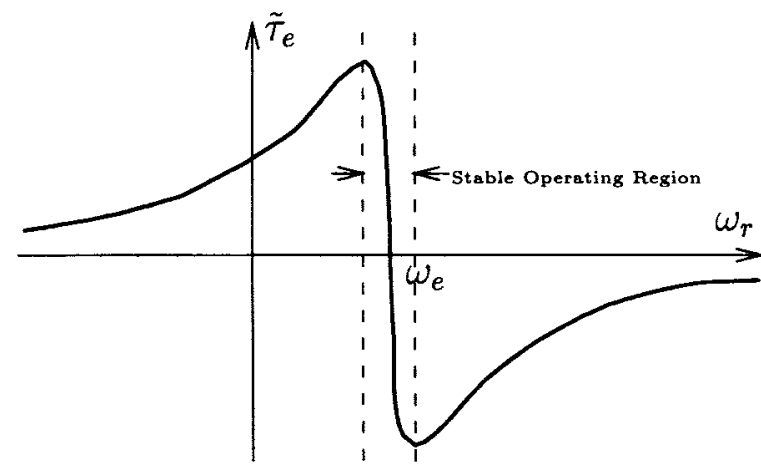

Fig. 1. Torque-speed curve.

We now analyze the "slow" mechanical error dynamics separately, with the view that the fast electrical dynamics have converged to the quasi-steady state given in (30). For example, we consider the electromagnetic torque error $\delta \tau_{e}$ to have reached a steady-state value $\delta \tilde{\tau}_{e}$. Using (13) and (30), we can write $\delta \tilde{\tau}_{e}$ as a function of the operating point of the machine (as characterized by $v_{s}^{e}, \omega_{e}$, and $\omega_{r}$ ), and the rotor speed error $\delta \omega_{r}$ :

$$
\begin{aligned}
\delta \tilde{\tau}_{e} & =\frac{M}{L_{r}}\left(\tilde{\hat{\vartheta}}_{s}^{e T} \mathbf{J} \tilde{\hat{\lambda}}_{r}^{e}-\tilde{\imath}_{s}^{e T} \mathbf{J} \tilde{\lambda}_{r}^{e}\right) \\
& =\frac{M}{L_{r}}\left(\delta \tilde{\imath}_{s}^{e T} \mathbf{J} \delta \tilde{\lambda}_{r}^{e}+\delta \tilde{v}_{s}^{e T} \mathbf{J} \tilde{\lambda}_{r}^{e}+\tilde{\imath}_{s}^{e T} \mathbf{J} \delta \tilde{\lambda}_{r}^{e}\right) \\
& =g_{\tau}\left(v_{s}^{e}, \omega_{e}, \omega_{r}\right) \delta \omega_{r}+O\left(\delta \omega_{r}^{2}\right) .
\end{aligned}
$$

The linear coefficient $g_{\tau}\left(v_{s}^{e}, \omega_{e}, \omega_{r}\right)$ is equivalent to $\partial \tilde{\tau}_{e} / \partial \omega_{r}$, the slope of the steady-state torque-speed curve for open-loop voltage excitation, as shown in Fig. 1. We note for future reference that $g_{\tau}\left(v_{s}^{e}, \omega_{e}, \omega_{r}\right)$ is negative in the open-loop stable operating region of the induction machine.

We now discuss the correction term $f_{c x}\left(\imath_{s}, \hat{\imath}_{s}, v_{s}^{e}, \omega_{e}\right)$. The formulation of this term can be understood by considering the locus of steady-state stator currents as a function of rotor speed, as shown in Fig. 2. From Fig. 2, it is evident that one can uniquely relate the steady-state stator current vector to the rotor speed. Furthermore, for a given stator voltage and excitation frequency, both $\imath_{s}^{e}$ and $\hat{\imath}_{s}^{e}$ will be on the same locus, with their positions depending on $\omega_{r}$ and $\hat{\omega}_{r}$, respectively. In order to develop the correction term we create vectors $\imath_{s}^{e}-\imath_{s x}$ and $\hat{\imath}_{s}^{e}-\imath_{s x}$, such as those shown in Figs. 3 and 4, where $\imath_{s x}$ is a vector in the stator current direct-quadrature plane the coordinates of which are geometrically enclosed in the arc formed by the stator current locus. There is a monotonic dependence between the angle $\theta$ between these two vectors and the rotor speed error. Instead of calculating $\theta$, however, we use the cross product of the two vectors to form our correction term:

$$
\begin{aligned}
f_{c x}\left(\imath_{s}, \hat{\imath}_{s}, v_{s}^{e}, \omega_{e}\right) & =\left(\imath_{s}^{e}-\imath_{s x}\right)^{T} \mathbf{J}\left(\hat{\imath}_{s}^{e}-\imath_{s x}\right) \\
& =\left\|\imath_{s}^{e}-\imath_{s x}\right\|\left\|\hat{\imath}_{s}^{e}-\imath_{s x}\right\| \sin \theta .
\end{aligned}
$$

Provided $|\theta|<180^{\circ}, f_{c x}\left(\tilde{\vartheta}_{s}, \tilde{\hat{\imath}}_{s}, v_{s}^{e}, \omega_{e}\right)$ has the same sign as $\theta$ and, therefore, the same sign as the rotor speed error $\delta \omega_{r}$. Hence, $f_{c x}\left(\tilde{\imath}_{s}, \tilde{\hat{\imath}}_{s}, v_{s}^{e}, \omega_{e}\right)$ can be used to correct estimated rotor speed.

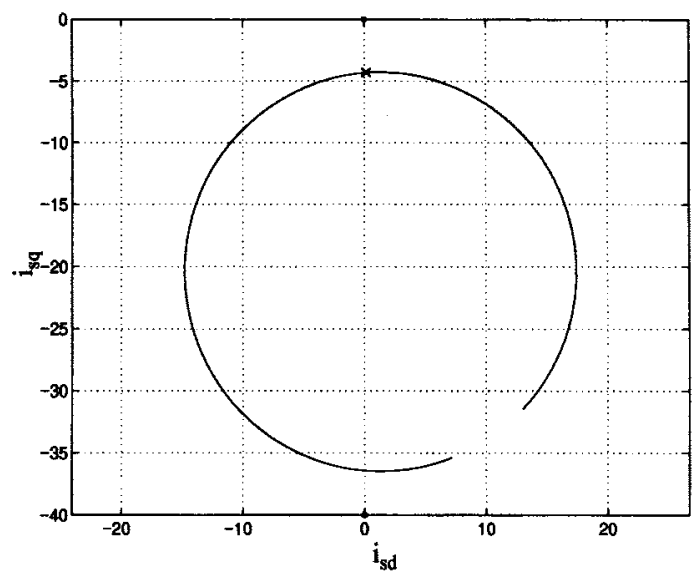

Fig. 2. Calculated stator current locus in electrical reference frame aligned with the stator voltage, as a function of rotor speed. $v_{s d}=190 \mathrm{~V}, f_{e}=60$ $\mathrm{Hz}$, typical motor parameters. The " $x$ " on the locus corresponds to zero slip frequency.

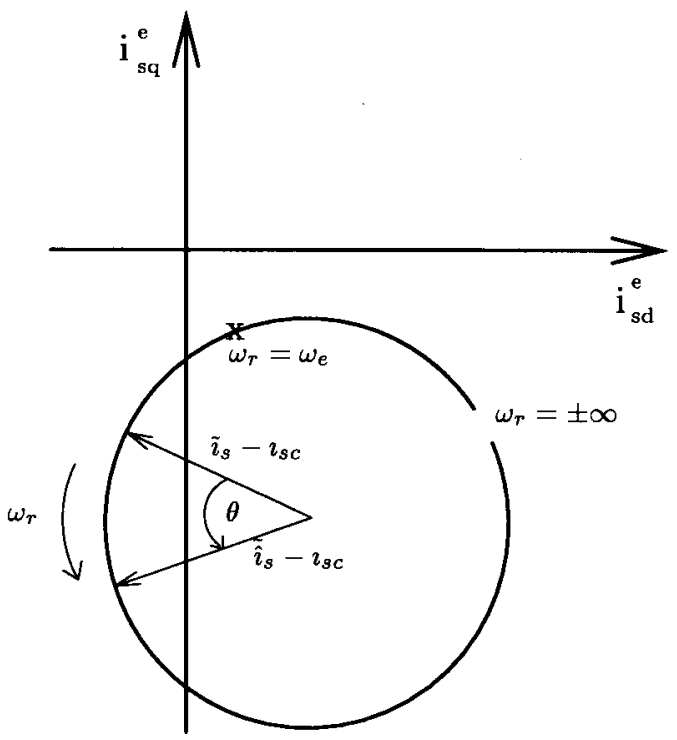

Fig. 3. Graphical description of correction term using coordinates corresponding to center of stator current locus.

We examine two particular choices for $\imath_{s x}$. One choice, $\imath_{s c}$, corresponds to the geometric center of the locus, as shown in Fig. 3:

$$
\imath_{s c}=\left[\begin{array}{c}
\frac{R_{s} L_{r}}{R_{s}^{2} L_{r}+L_{s} \sigma^{2} \omega_{e}^{2}} \\
-\left(\sigma^{2}+\frac{M^{2}}{2}\right) \omega_{e} \\
\frac{R_{s}^{2} L_{r}+L_{s} \sigma^{2} \omega_{e}^{2}}{v_{s d}} .
\end{array} v^{e}\right.
$$

The other choice, $\imath_{s \infty}$, corresponds to the theoretical steadystate stator current at infinite rotor speed, as shown in Fig. 4:

$$
\imath_{s \infty}=\left(R_{s} \mathbf{I}+\frac{\sigma^{2}}{L_{r}} \omega_{e} \mathbf{J}\right)^{-1} v_{s}^{e} .
$$

Both choices for $\imath_{s x}$ have features making them desirable. It is intuitively clear from Figs. 3 and 4 that the choice of $\imath_{s c}$ will yield a greater sensitivity to the rotor speed error than 


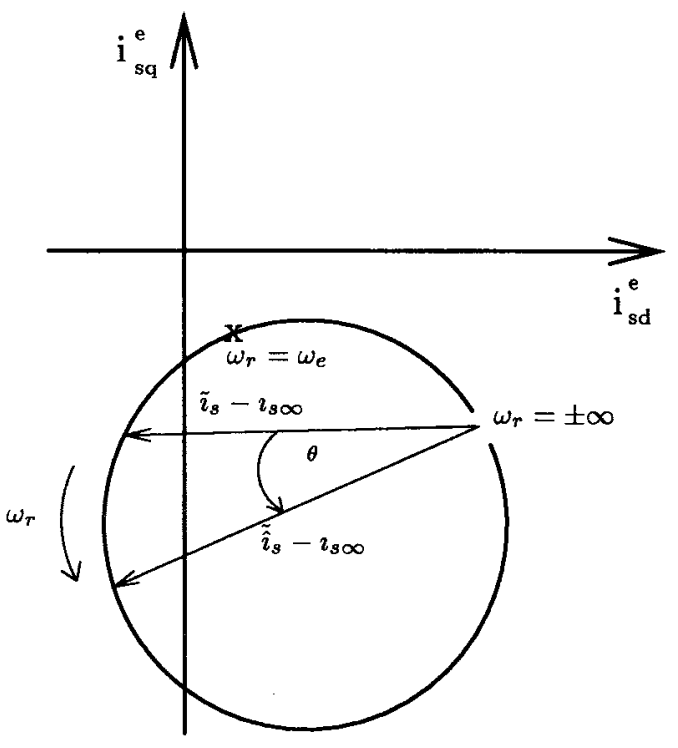

Fig. 4. Graphical description of correction term using coordinates corresponding to stator current at infinite rotor speed.

$\imath_{s \infty}$. However, $\imath_{s c}$ has the disadvantage that, for extremely large speed errors, $|\theta|$ can become greater than $180^{\circ}$, in which case the sign of the cross-product term differs from the sign of the rotor speed error. The vectors $\tilde{\imath}_{s}^{e}-\imath_{s \infty}$ and $\hat{\tilde{\imath}}_{s}^{e}-\imath_{s \infty}$ are never more than $180^{\circ}$ apart and, so, $f_{c \infty}\left(\tilde{\imath}_{s}, \tilde{\hat{\imath}}_{s}, v_{s}^{e}, \omega_{e}\right)$ is an effective correction term for all rotor speed errors.

In electrical steady state, $f_{c x}\left(\tilde{\imath}_{s}, \tilde{\hat{\imath}}_{s}, v_{s}^{e}, \omega_{e}\right)$ can also be written as a function of the operating point and the speed error, using (13) and (30) and the property $x^{T} \mathbf{J} x=0$

$$
\begin{aligned}
f_{c x}\left(\imath_{s}, \hat{\imath}_{s}, v_{s}^{e}, \omega_{e}\right) & =\left(\tilde{\imath}_{s}^{e}-\imath_{s x}\right)^{T} \mathbf{J}\left(\tilde{\imath}_{s}^{e}-\imath_{s x}\right) \\
& =\left(\tilde{\imath}_{s}^{e}-\imath_{s x}\right)^{T} \mathbf{J}\left(\delta \tilde{\imath}_{s}+\tilde{\imath}_{s}^{e}-\imath_{s x}\right) \\
& =\left(\tilde{\imath}_{s}^{e}-\imath_{s x}\right)^{T} \mathbf{J} \delta \tilde{\imath}_{s} \\
& =h_{c x}\left(v_{s}^{e}, \omega_{r}, \omega_{e}\right) \delta \omega_{r}+O\left(\delta \omega_{r}^{2}\right) .
\end{aligned}
$$

As we are assuming small-signals, we neglect the higher order terms of $\delta \omega_{r}$ and focus on the linear component $h_{c x}\left(v_{s}^{e}, \omega_{r}, \omega_{e}\right) \delta \omega_{r}$. Analytical expressions for $h_{c c}$ and $h_{c \infty}$ are presented in (36) and (37) at the bottom of the page. Inspection of the above expressions reveals that $h_{c c}>0$ and $h_{c \infty}>0$ for all possible operating points, with the exception of dc excitation. In the case of dc excitation, the stator current locus collapses to a single point $v_{s} / R_{s}$, hence, $\imath_{s}^{e}=\hat{\imath}_{s}^{e}=\imath_{s x}$ and the correction term vanishes. As discussed in Section IV, all speed estimation schemes that use only stator voltage and current measurements and assume the smooth-airgap model have this same limitation. That is, the stator current and voltage do not contain any information about the rotor speed at dc.

Fig. 5 presents normalized three-dimensional (3-D) plots of $h_{c c}, h_{c \infty}$, and $g_{\tau}$ for a range of operating points, characterized
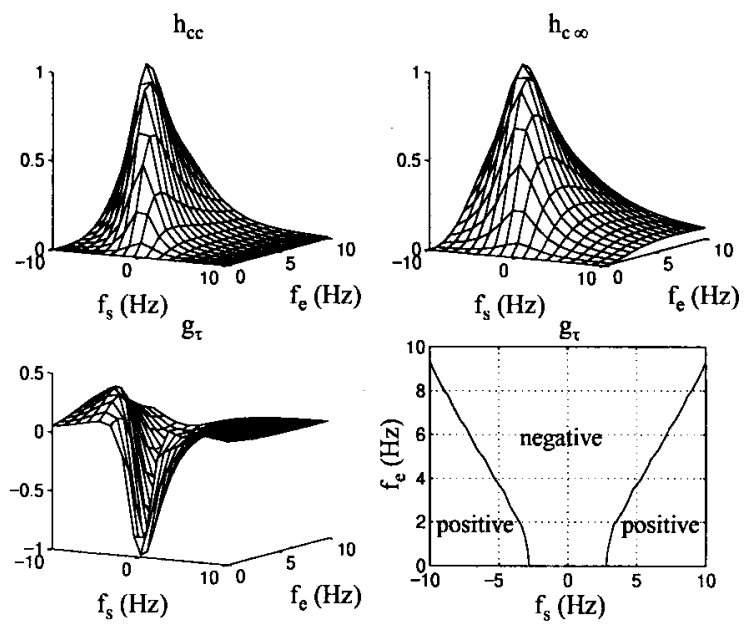

Fig. 5. Normalized 3-D plots of $h_{c c}, h_{c \infty}$, and $g_{\tau}$ as functions of electrical and slip frequency, with a contour plot of $g_{\tau}$ demarcating regions of positive and negative sign.

by the electrical and slip frequencies. Also shown is a contour plot of $g_{\tau}$ demarcating its regions of positive and negative sign. Recall that $g_{\tau}$ is the slope of the steady-state torque-speed curve for open-loop voltage excitation.

Assuming that the electrical error dynamics have settled to their quasi-steady-state value, we can write the mechanical dynamics in linearized form:

$$
\begin{aligned}
{\left[\begin{array}{c}
\dot{\delta \omega_{r}} \\
\dot{\delta \tau_{\ell}}
\end{array}\right] } & =\frac{1}{H} \mathbf{A}_{s}\left[\begin{array}{l}
\delta \omega_{r} \\
\delta \tau_{\ell}
\end{array}\right], \\
\mathbf{A}_{s} & =\left[\begin{array}{cr}
\left(g_{\tau}-K_{\omega} h_{c x}-B\right) & -1 \\
K_{\tau} h_{c x} & 0
\end{array}\right] .
\end{aligned}
$$

Provided the induction machine is operating in the openloop stable operating range [i.e., $g_{\tau}\left(v_{s}^{e}, \omega_{r}, \omega_{e}\right)<0$ ], it can be easily shown that the matrix $\mathbf{A}_{s}$ is exponentially stable if $h_{c x}\left(v_{s}^{e}, \omega_{r}, \omega_{e}\right)>0$, stable if $h_{c x}\left(v_{s}^{e}, \omega_{r}, \omega_{e}\right)=0$, and unstable if $h_{c x}\left(v_{s}^{e}, \omega_{r}, \omega_{e}\right)<0$.

In the following, we use the term $1 / H$ to conceptually separate the time scales of the electrical and mechanical dynamics. In other words, provided the moment of inertia $H$ is sufficiently large, the mechanical dynamics will be slow enough with respect to the electrical dynamics to validate the independent analysis.

We have now shown stability of both the fast and slow error dynamics. To prove stability of the entire system we use Corollary 3.1 of Kokotovic [6]. This corollary guarantees asymptotic stability of the error dynamics provided the following are true.

1) $\mathbf{A}_{f}$ and $\mathbf{A}_{s}$ are exponentially stable.

2) The "slow" dynamics are "slow" enough, i.e., $1 / H$ is less than some upper bound $\epsilon_{0}$.

$$
\begin{aligned}
& h_{c c}=\left.\frac{\partial f_{c c}}{\partial\left(\delta \omega_{r}\right)}\right|_{\delta \omega_{r}=0}=\frac{M^{4} \omega_{e}^{2} R_{r} v_{s d}^{e 2}}{2\left(R_{s}^{2} L_{r}+\omega_{e}^{2} L_{s} \sigma^{2}\right)\left[\left(\omega_{s} L_{r} R_{s}+\omega_{e} L_{s} R_{r}\right)^{2}+\left(R_{s} R_{r}-\omega_{e} \omega_{s} \sigma^{2}\right)^{2}\right]} \\
& h_{c \infty}=\left.\frac{\partial f_{c \infty}}{\partial\left(\delta \omega_{r}\right)}\right|_{\delta \omega_{r}=0}=\frac{M^{4}\left(\omega_{e}^{2} \sigma^{2} L_{s}+R_{s}^{2} L_{r}\right) \omega_{e}^{2} R_{r}^{3} v_{s d}^{e 2}}{\left[\left(R_{s} R_{r}-\omega_{e} \omega_{s} \sigma^{2}\right)^{2}+\left(R_{s} L_{r} \omega_{s}+R_{r} L_{s} \omega_{e}\right)^{2}\right]^{2}\left(\omega_{e}^{2} \sigma^{4}+R_{s}^{2} L_{r}^{2}\right)}
\end{aligned}
$$




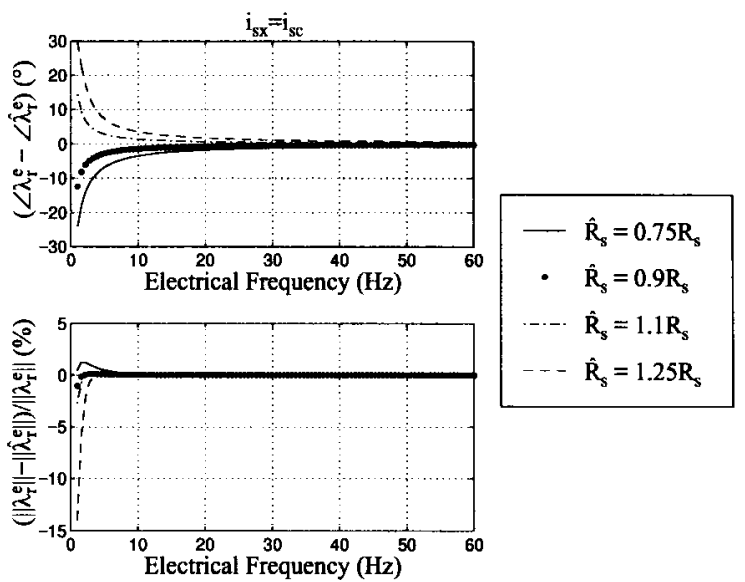

Fig. 6. Steady-state observer rotor flux error due to deviations in $R_{s}$ using correction term $f_{c c}\left(\imath_{s}, \hat{\imath}_{s}, v_{s}^{e}, \omega_{e}\right), \omega_{s}=0$.

The upper bound $\epsilon_{0}$ is difficult to determine. For a given steady-state operating point, we can derive a lower bound for $\epsilon_{0}$ [1, eq. (2.18)]. However, as this is only a lower bound for $\epsilon_{0}$, it is desirable to determine the gains through simulation or experimentation, in order to achieve satisfactory performance.

\section{PARAMETER Sensitivity}

This section analyzes the effect of errors in the observer parameters on the observer's performance. As many fieldoriented torque-control schemes used in practice are rotor-flux based, we analyze the effect of parameter deviation on the steady-state rotor flux angle and magnitude. Analysis is limited to operating points where the slip frequency is set to onetenth the value of the electrical frequency. We first calculate $\tilde{\lambda}_{r}^{e}$ for a given operating point. We then alter a parameter in the observer model and calculate $\tilde{\hat{\lambda}}_{r}$ such that the correction term is identically zero, i.e., $\left(\hat{\imath}_{s}^{e}-\imath_{s x}\right)^{T} \mathbf{J}\left(\tilde{\imath}_{s}^{e}-\imath_{s x}\right)=0$ is satisfied. This corresponds to the condition when the observer has converged to its steady-state value.

Figs. 6-8 present angular and magnitude errors of the observer rotor flux due to deviations in the parameters $R_{s}, M$, and $L_{\ell s}$, respectively. The correction term makes the rotor flux completely insensitive to $R_{r}$ and highly insensitive to $L_{\ell r}$ in steady state and, therefore, sensitivity to these parameters is not presented in this paper. In this analysis, we chose $\imath_{s x}=\imath_{s c}$. The sensitivity analysis for $\imath_{s \infty}$ is not presented, as it is similar to that of $\imath_{s c}$.

It is apparent from Figs. 6-8 that the observer is highly sensitive to deviations in $R_{s}$ and $M$ at low electrical frequencies. As stator resistance can vary markedly due to temperature, it is desirable to either include a temperature-compensated model for $R_{s}$ or implement a stator resistance estimation scheme. The parameter $M$ can also vary due to magnetic saturation. However, we note that the above observer can readily incorporate a nonlinear magnetics model, such as the one presented in [10].

\section{IMPLEMENTATION}

We implemented the observer on a $90-\mathrm{MHz}$ personal computer which samples stator voltages and currents and com-

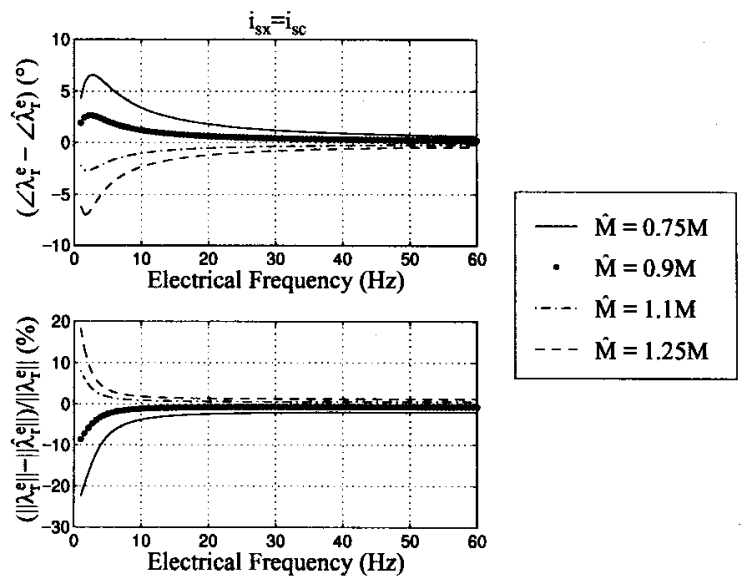

Fig. 7. Steady-state observer rotor flux error due to deviations in $M$ using correction term $f_{c c}\left(\imath_{s}, \hat{\imath}_{s}, v_{s}^{e}, \omega_{e}\right), \omega_{s}=0$.

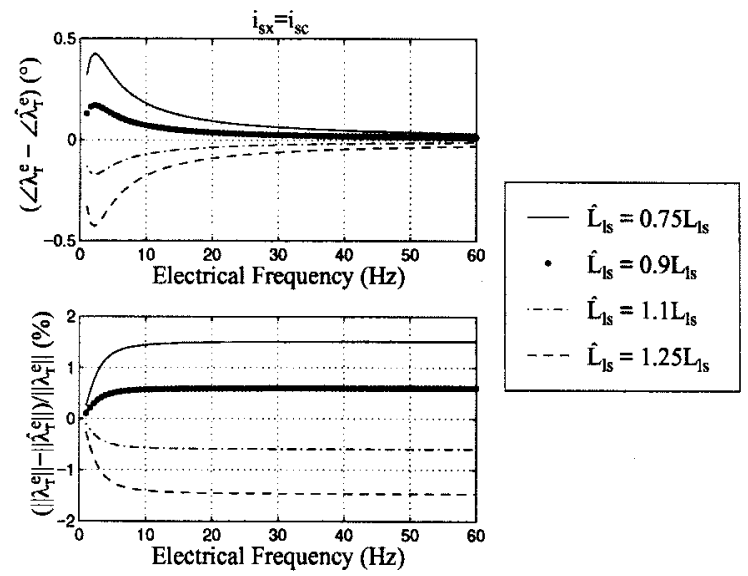

Fig. 8. Steady-state observer rotor flux error due to deviations in $L_{\ell s}$ using correction term $f_{c c}\left(\imath_{s}, \hat{\imath}_{s}, v_{s}^{e}, \omega_{e}\right), \omega_{s}=0$.

mands stator voltage values with sampling time $T_{s}=100$ $\mu \mathrm{s}$. The stator current and voltage measurements are filtered with second-order $1-\mathrm{kHz}$ antialiasing filters. The computer numerically integrates (23)-(25) using the third-order Adams-Bashforth method [11]. In order to generate the correction term $f_{c x}\left(\imath_{s}, \hat{s}_{s}, v_{s}^{e}, \omega_{e}\right)$, the computer must transform the motor and observer stator currents into the electrical reference frame with respect to the stator voltage. This is done using the transformation

$$
\imath_{s}^{e}=\frac{1}{\left\|v_{s}\right\|}\left[\begin{array}{rr}
v_{s d} & v_{s q} \\
-v_{s q} & v_{s d}
\end{array}\right] \imath_{s} .
$$

Knowledge of the electrical frequency is also necessary to determine $f_{c x}\left(v_{s}, \hat{\imath}_{s}, v_{s}^{e}, \omega_{e}\right)$. We calculate electrical frequency using a formula presented in [12]

$$
\omega_{e}=\frac{\dot{v}_{s}^{T} \mathbf{J} v_{s}}{\left\|v_{s}\right\|^{2}}
$$

where $\dot{v}_{s}$ is calculated using a band-limited numerical differentiation scheme with a bandwidth of $1000 \mathrm{rad} / \mathrm{s}$. The limited bandwidth does not present a problem, as the electrical frequency calculation is used only in the slow dynamics.

We tested the observer by implementing torque steps at low rotor speeds. Torque commands are generated through 

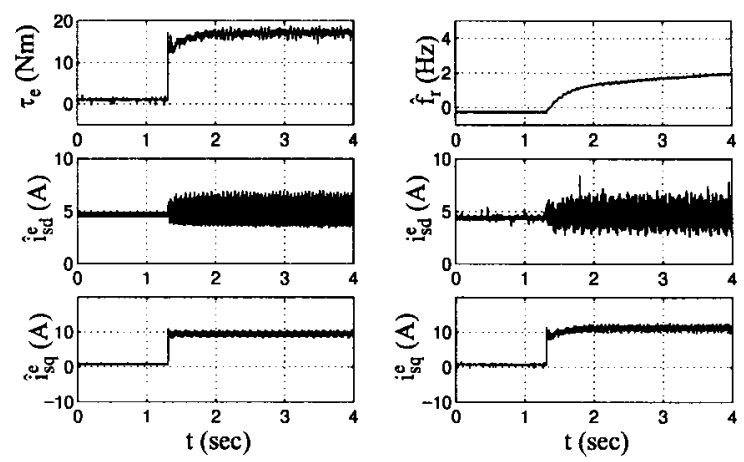

Fig. 9. Torque step from 0 to $18 \mathrm{~N} \cdot \mathrm{m}$. Plots, going clockwise from the top left, are estimated torque, observer rotor speed, direct and quadrature motor stator current, and quadrature and direct observer stator current. Currents are displayed in the electrical reference frame with respect to rotor flux.
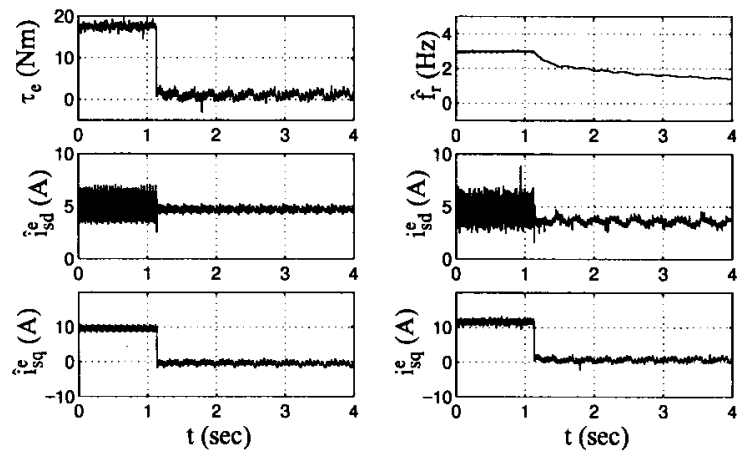

Fig. 10. Torque step from 18 to $0 \mathrm{~N} \cdot \mathrm{m}$. Plots as in Fig. 9.
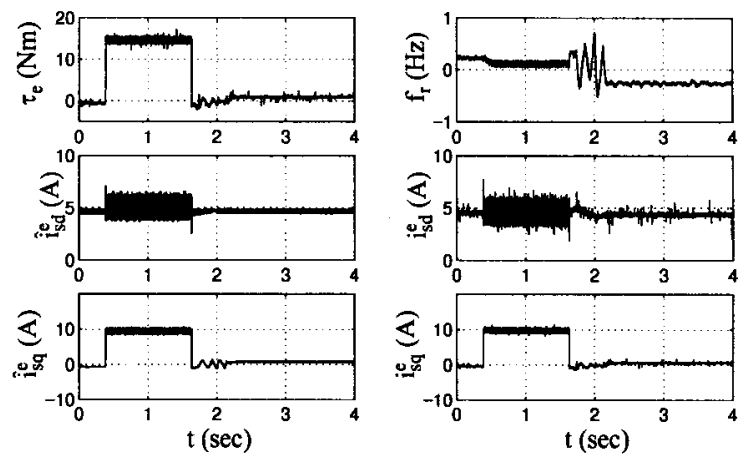

Fig. 11. Torque step from no torque to rated torque, locked rotor. Plots as in Fig. 9.

control of the direct and quadrature components of stator flux in the rotor flux reference frame, as presented in [12]. Figs. 9 and 10 show the result of torque step commands with a $40-\mathrm{kW}$ separately excited dynamometer providing resistive loading for the induction machine. Fig. 11 shows the results of a locked-rotor test, with a torque step from zero to rated torque. Although the rotor was locked, this information was not supplied to the observer. We estimate torque using the cross product of estimated stator flux and measured stator current, i.e., $3 \hat{\lambda}_{s}^{T} \mathbf{J} l_{s}$. Also presented in the plots are estimated rotor speed and observer and motor stator currents in the electrical reference frame with respect to the rotor flux.

For these experiments, we use the correction term $f_{c c}\left(\imath_{s}, \hat{v}_{s}, v_{s}^{e}, \omega_{e}\right)$. In order to reduce the effect of the induction machine operating point on the correction term, we choose variable gains $K_{\omega}=1000 /\left(\left\|\imath_{s}^{e}-\imath_{s x}\right\| \| \hat{\imath}_{s}^{e}-\right.$ $\left.\imath_{s x} \|+0.001\right)$ and $K_{\tau}=1 /\left(\left\|\imath_{s}^{e}-\imath_{s x}\right\|\left\|\hat{\imath}_{s}^{e}-\imath_{s x}\right\|+0.001\right)$. If we neglect the small constant in the denominator, which is included to avoid a singularity at dc excitation, we have $K_{\omega} f_{c c}\left(\imath_{s}, \hat{\imath}_{s}, v_{s}\right)=1000 \sin \theta$ and $K_{\tau} f_{c c}\left(v_{s}, \hat{\imath}_{s}, v_{s}\right)=$ $\sin \theta$.

The motor used in the experiments is a three-phase fourpole wound-rotor induction machine rated at $3 \mathrm{hp}, 220 \mathrm{~V}$ line-to-line, and $9 \mathrm{~A}$. The motor is driven by a commercial pulsewidth-modulated (PWM) insulated gate bipolar transistor (IGBT) inverter rated at $36 \mathrm{~A}$ and $460 \mathrm{~V}$. The microprocessor control provided in the inverter was replaced with custom hardware that directly accesses the gate drive modules. This hardware consists of a three-phase $15-\mathrm{kHz}$ PWM modulator that interfaces with two-axis command voltages supplied by the personal computer. Hall-effect sensors within the inverter measure the stator currents and $100: 1$ voltage probes measure the stator voltages.

The control scheme is shown to work well, allowing stable torque steps at low and zero electrical frequencies. A brief disturbance can be seen in the locked-rotor test (Fig. 11) as the commanded torque changes from rated torque to zero torque. This is because the electrical frequency changes from a positive number to zero, where the system becomes unobservable. Note, however, that the observer/machine system eventually settles.

\section{ANALYSIS OF ANOTHER FULL-ORDER OBSERVER}

The two-time-scale approach can also be used to analyze other speed-sensorless control schemes. For example, we consider the full-order adaptive observer presented in [2]. This observer is a convenient choice for analysis, as it uses the same model for electrical dynamics presented in this paper [see (23)]. Although [2] discusses the use of feedback injection into the electrical dynamics, this is not implemented in their experiments and, so, we do not include this feedback injection in the following analysis.

For the mechanical dynamics, [2] assumes constant rotor speed and estimates this speed using the following correction term:

$$
\dot{\hat{\omega}}_{r}=-\hat{\lambda}_{r}^{T} \mathbf{J}\left(\hat{\imath}_{s}-\imath_{s}\right) .
$$

By assuming that the electrical error variables have converged to the quasi-steady state of (30), we can write the rotor speed error dynamics in terms of the rotor speed error and the operating point of the machine (13):

$$
\begin{aligned}
\dot{\delta} \dot{\omega}_{r} & =-\hat{\hat{\lambda}}_{r}^{e T} \mathbf{J} \delta \tilde{\imath}_{s}^{e} \\
& =-h_{c k}\left(v_{s}, \omega_{r}, \omega_{e}\right) \delta \omega_{r}+O\left(\delta \omega_{r}^{2}\right) .
\end{aligned}
$$

Once again, we assume small signals and, therefore, neglect the higher order terms of (43). Based on the assumptions of singular perturbation theory, the rotor speed error dynamics are locally asymptotically stable if $h_{c k}\left(v_{s}^{e}, \omega_{r}, \omega_{e}\right)>0$ and unstable if $h_{c k}\left(v_{s}^{e}, \omega_{r}, \omega_{e}\right)<0$

Fig. 12 shows a normalized 3-D plot and contour plot of $h_{c k}\left(v_{s}^{e}, \omega_{r}, \omega_{e}\right)$ using the motor parameters presented in this 

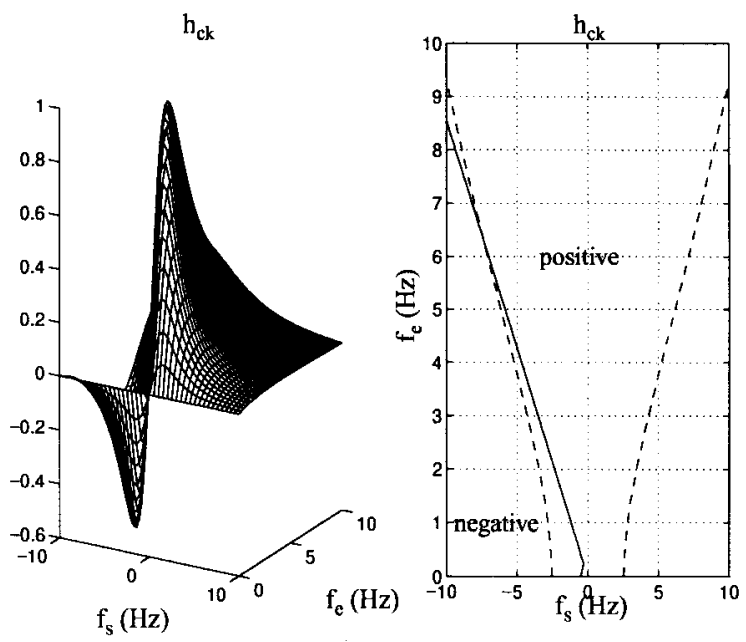

Fig. 12. Normalized 3-D plot of $h_{c k}$ and contour plots of $h_{c k}$ (solid line) and $g_{\tau}$ (dashed line) demarcating regions of positive and negative sign. Regions of positive and negative sign of $h_{c k}$ are specified. Typical motor parameters.

paper. The contour plot of $g_{\tau}\left(v_{s}^{e}, \omega_{r}, \omega_{e}\right)$ is superimposed over $h_{c k}$ for reference.

Although $h_{c k}\left(v_{s}^{e}, \omega_{r}, \omega_{e}\right)>0$ for a wide range of operating conditions, there exists a range of operating points in the generating region $\left(\omega_{s}<0\right)$ where it becomes negative and, hence, the observer does not converge under the assumptions of the singular perturbation theory. We note, however, that only a limited portion of this unstable range overlaps with the stable operating region of the induction machine, as shown in Fig. 12.

\section{CONCLUSION}

We have shown singular perturbation theory to be an effective tool in the analysis of induction machine observers. Using the assumption of separate time scales, a full-order observer has been designed that converges at all operating points, with the exception of dc excitation. Although the technique was used in this paper to estimate rotor speed, the same approach could be used to estimate other parameters of an induction machine that are slowly varying, such as stator resistance. Further study is warranted to explore the usefulness of this approach.

\section{APPENDIX}

We now seek to prove that the vector $a$ shown in (22) satisfies (21), i.e., we seek to show that there exists a combination of the columns of $\mathcal{C}_{O}$ that sum to zero:

$$
a_{1} \mathcal{C}_{o 1}+a_{2} \mathcal{C}_{o 2}+a_{3} \mathcal{C}_{o 3}+a_{4} \mathcal{C}_{o 4}+a_{5} \mathcal{C}_{o 5}+a_{6} \mathcal{C}_{o 6}=\mathbf{0}
$$

where the $a_{i}$ are the components of the vector $a$. Inspection of $\mathbf{C}_{\ell}$ (19) (the first two columns of which are linearly independent and the remaining columns of which contain zeros) reveals that any such combination cannot contain the first two columns, hence, $a_{1}=a_{2}=0$. We therefore focus on linear combinations of the last four columns.

Next we look at $\mathbf{C}_{\ell} \mathbf{A}_{\ell}$ (see (45) at the bottom of the page). From this, we can surmise that $a_{3}, a_{4}$, and $a_{5}$ must satisfy the following condition:

$$
\left(\frac{M R_{r}}{\sigma^{2} L_{r}} \mathbf{I}-\frac{M}{\sigma^{2}} \omega_{r} \mathbf{J}\right)\left[\begin{array}{l}
a_{3} \\
a_{4}
\end{array}\right]-a_{5} \frac{M}{\sigma^{2}} \mathbf{J} \lambda_{r}^{e}=\mathbf{0}_{2 \times 1} .
$$

Without loss of generality, we can set $a_{\tilde{5}}=1$, as scalar multiples of the $a_{i}$ will still satisfy (21). We can then solve for $a_{3}$ and $a_{4}$ :

$$
\left[\begin{array}{l}
a_{3} \\
a_{4}
\end{array}\right]=\left(\frac{M R_{r}}{\sigma^{2} L_{r}} \mathbf{I}-\frac{M}{\sigma^{2}} \omega_{r} \mathbf{J}\right)^{-1} \frac{M}{\sigma^{2}} \mathbf{J} \lambda_{r}^{e} .
$$

We note that $a_{3}, a_{4}$, and $a_{5}$ are unique to within a scalar multiple. It remains to determine $a_{6}$, if it exists. We now consider $\mathbf{C}_{\ell} \mathbf{A}_{\ell}^{2}$. The actual matrix, however, is quite large and complex, hence, we shall break it into columns in order to display it effectively

$$
\begin{aligned}
& \left(\mathbf{C}_{\ell} \mathbf{A}_{\ell}^{2}\right)_{1,2}= \\
& \left(\frac{R_{s}^{2} L_{r}^{2}+2 R_{s} R_{r} M^{2}}{\sigma^{4}}+\frac{R_{r} M^{4}}{\sigma^{4} L_{r}^{2}}+\frac{M^{2} R_{r}^{2}}{\sigma^{2} L_{r}^{2}}-\omega_{e}^{2}\right) \mathbf{I} \\
& \quad+\left(\frac{2 \omega_{e} R_{s} L_{r}}{\sigma^{2}}+\frac{2 \omega_{e} R_{r} M^{2}}{\sigma^{2} L_{r}}-\frac{M^{2} \omega_{r} R_{r}}{\sigma^{2} L_{r}}\right) \mathbf{J} \\
& \quad+\frac{M}{\sigma^{2} L_{r} H} \lambda_{r}^{e} \lambda_{r}^{e T} \\
& \left(\mathbf{C}_{\ell} \mathbf{A}_{\ell}^{2}\right)_{3,4}= \\
& \quad\left(-\frac{M R_{r} R_{s}}{\sigma^{4}}-\frac{M^{3} R_{r}^{2}}{\sigma^{4} L_{r}^{2}}-\frac{2 \omega_{e} \omega_{r} M}{\sigma^{2}}-\frac{R_{r}^{2} M}{\sigma^{2} L_{r}^{2}}+\frac{M \omega_{r}^{2}}{\sigma^{2}}\right) \mathbf{I} \\
& \quad+\left(-\frac{2 \omega_{e} R_{r} M}{\sigma^{2} L_{r}}+\frac{\omega_{r} L_{r} M R_{s}}{\sigma^{4}}+\frac{R_{r} M^{3} \omega_{r}}{\sigma^{4} L_{r}}\right. \\
& \left.\quad+\frac{2 \omega_{r} M R_{r}}{\sigma^{2} L_{r}}\right) \mathbf{J}+\frac{M^{2}}{\sigma^{2} L_{r} H}\left(\mathbf{J} \lambda_{r}^{e}\right)\left(\mathbf{J}_{s}^{e}\right)^{T} \\
& \left(\mathbf{C}_{\ell} \mathbf{A}_{\ell}^{2}\right)_{5}= \\
& \frac{M}{\sigma^{2}}\left[-\left(\omega_{e}-\omega_{r}\right) \mathbf{I}+\left(\frac{R_{s} L_{r}}{\sigma^{2}}+\frac{R_{r} M^{2}}{\sigma^{2} L_{r}}+\frac{R_{r}}{L_{r}}+B\right) \mathbf{J}\right] \lambda_{r}^{e} \\
& \left(\mathbf{C}_{\ell} \mathbf{A}_{\ell}^{2}\right)_{6}=\frac{M}{\sigma^{2} H} \mathbf{J} \lambda_{r}^{e} .
\end{aligned}
$$

To determine if $a_{6}$ exists and, if so, its value, we first find the result of the sum $a_{3}\left(\mathbf{C}_{\ell} \mathbf{A}_{\ell}^{2}\right)_{3}+a_{4}\left(\mathbf{C}_{\ell} \mathbf{A}_{\ell}^{2}\right)_{4}+a_{5}\left(\mathbf{C}_{\ell} \mathbf{A}_{\ell}^{2}\right)_{5}$, which we calculate using (47) and (51):

$$
\begin{aligned}
a_{3}\left(\mathbf{C}_{\ell} \mathbf{A}_{\ell}^{2}\right)_{3}+a_{4}\left(\mathbf{C}_{\ell} \mathbf{A}_{\ell}^{2}\right)_{4}+a_{5}\left(\mathbf{C}_{\ell} \mathbf{A}_{\ell}^{2}\right)_{5} \\
=\left(\mathbf{C}_{\ell} \mathbf{A}_{\ell}^{2}\right)_{3,4}\left[\begin{array}{l}
a_{3} \\
a_{4}
\end{array}\right]+\left(\mathbf{C}_{\ell} \mathbf{A}_{\ell}^{2}\right)_{5} \\
=\frac{M}{\sigma^{2}}\left\{\omega_{e} \mathbf{I}+\left[B+\frac{M}{\left(R_{r}^{2}+\omega_{r}^{2} L_{r}^{2}\right) H}\right.\right. \\
\\
\left.\left.\cdot\left[z_{s}^{e T}\left(R_{r} \mathbf{I}+\omega_{r} L_{r} \mathbf{J}\right) \lambda_{r}^{e}\right]\right] \mathbf{J}\right\} \lambda_{r}^{e} .
\end{aligned}
$$

$$
\mathbf{C}_{\ell} \mathbf{A}_{\ell}=\left[-\frac{R_{s} L_{r}^{2}+R_{r} M^{2}}{\sigma^{2} L_{r}} \mathbf{I}-\omega_{e} \mathbf{J} \frac{M R_{r}}{\sigma^{2} L_{r}} \mathbf{I}-\frac{M}{\sigma^{2}} \omega_{r} \mathbf{J}-\frac{M}{\sigma^{2}} \mathbf{J} \lambda_{r}^{e} \mathbf{0}\right] .
$$


If the system is unobservable, then there must exist a scalar $a_{6}$ such that

$$
a_{3}\left(\mathbf{C}_{\ell} \mathbf{A}_{\ell}^{2}\right)_{3}+a_{4}\left(\mathbf{C}_{\ell} \mathbf{A}_{\ell}^{2}\right)_{4}+a_{5}\left(\mathbf{C}_{\ell} \mathbf{A}_{\ell}^{2}\right)_{5}=-a_{6}\left(\mathbf{C}_{\ell} \mathbf{A}_{\ell}^{2}\right)_{6}
$$

or

$$
\begin{aligned}
a_{6} \frac{M}{\sigma^{2} H} \mathbf{J} \lambda_{r}^{e}= & -\frac{M}{\sigma^{2}}\left(\omega_{e} \mathbf{I}+\left\{B+\frac{M}{\left(R_{r}^{2}+\omega_{r}^{2} L_{r}^{2}\right) H}\right.\right. \\
& \left.\left.\cdot\left[\vartheta_{s}^{e T}\left(R_{r} \mathbf{I}+\omega_{r} L_{r} \mathbf{J}\right) \lambda_{r}^{e}\right]\right\} \mathbf{J}\right) \lambda_{r}^{e} .
\end{aligned}
$$

Note that the vector on the left-hand side of (54) is orthogonal to $\lambda_{r}^{e}$, whereas the right-hand side contains components parallel and orthogonal to $\lambda_{r}^{e}$. Therefore, for $a_{6}$ to exist, the component of the right-hand side parallel to $\lambda_{r}^{e}$ must disappear (assuming that $\lambda_{r}^{e}$ is nonzero, as previously stated). This occurs if and only if $\omega_{e}=0$. Hence, $\omega_{e}=0$ is a requirement for the system to be unobservable.

If $\omega_{e}=0$, then we can determine $a_{6}$ from (54):

$$
a_{6}=-\left\{B H+\frac{M}{\left(R_{r}^{2}+\omega_{r}^{2} L_{r}^{2}\right)}\left[\imath_{s}^{e T}\left(R_{r} \mathbf{I}+\omega_{r} L_{r} \mathbf{J}\right) \lambda_{r}^{e}\right]\right\}
$$

It can then be shown (although the calculations are extensive and were performed using MAPLE) that, provided $\omega_{e}=0$,

$$
\mathcal{C}_{o} a=\mathbf{0}_{12 \times 1}
$$

hence, the smooth-airgap induction machine model is locally observable everywhere, except for dc excitation.

\section{ACKNOWLEDGMENT}

The authors would like to thank T.-Y. Chen for her efforts in proofreading this manuscript.

\section{REFERENCES}

[1] H. Nakano and I. Takahashi, "Sensorless field-oriented control of an induction motor using an instantaneous slip frequency estimation method," in Conf. Rec. PESC'88, 1988, pp. 847-854.

[2] H. Kubota, K. Matsuse, and T. Nakano, "DSP-based speed adaptive flux observer of induction motor," IEEE Trans. Ind. Applicat., vol. 29, pp. 344-348, Mar./Apr. 1993.

[3] Y. Kim, S. Sul, and M. Park, "Speed sensorless vector control of induction motor using extended kalman filter," IEEE Trans. Ind. Applicat., vol. 30, pp. 1225-1233, Sept./Oct. 1994.

[4] F. Peng and T. Fukao, "Robust speed identification for speed-sensorless vector control of induction motors," IEEE Trans. Ind. Applicat., vol. 30, pp. 1234-1240, Sept./Oct. 1994.

[5] N. Rubin, R. Harley, and G. Diana, "Evaluation of various slip estimation techniques for an induction machine operating under field-oriented control conditions," IEEE Trans. Ind. Applicat., vol. 28, pp. 1367-1375, Nov./Dec. 1992.

[6] P. Kokotovic, H. K. Khalil, and J. O'Reilly, Singular Perturbation Methods in Control: Analysis and Design. New York: Academic, 1986.

[7] M. Velez-Reyes and G. Verghese, "Developing reduced order electrica machine models using participation factors," Model. Simul. Syst., vol. 3, no. 1, pp. 333-335, 1989.

[8] T. Kailath, Linear Systems. Englewood Cliffs, NJ: Prentice-Hall, 1980

[9] G. Verghese and S. Sanders, "Observers for flux estimation in induction machines," IEEE Trans. Ind. Electron., vol. 35, pp. 85-94, Feb. 1988.

[10] C. R. Sullivan and S. R. Sanders, "Models for induction machines with magnetic saturation of the main flux path," in Conf. Rec. IEEE-IAS Annu. Meeting, 1992, vol. 1, pp. 123-31.

[11] J. D. Lambert, Numerical Methods for Ordinary Differential Systems: The Initial Value Problem. New York: Wiley, 1991.

[12] C. Sullivan, C. Kao, B. Acker, and S. Sanders, "Control systems for induction machines with magnetic saturation," IEEE Trans. Ind. Electron., vol. 43, pp. 142-152, Feb. 1996.

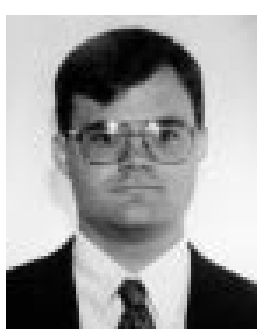

machinery.
Heath Hofmann (S'97) was born in the Texas Panhandle in 1969. He received the B.S. degree in electrical engineering from the University of Texas, Austin, in 1992 and is currently working toward the Ph.D. degree at the University of California, Berkeley.

He has published technical papers on power distribution systems, induction machine control, and the design of synchronous reluctance machines. His research interests include the simulation, design, and control of power electronics circuits and electrical

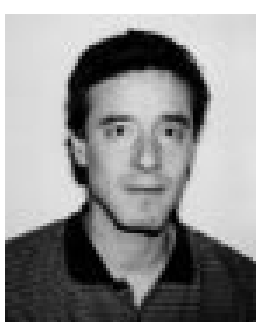

Seth R. Sanders (M'87) received B.S. degrees in electrical engineering and physics and the M.S and $\mathrm{Ph} . \mathrm{D}$. degrees in electrical engineering from Massachusetts Institute of Technology, Cambridge, in 1981, 1985, and 1989, respectively.

Prior to working toward the M.S. degree, he was a Design Engineer with the Honeywell Test Instruments Division, Denver, CO. Since 1989, he has been a Faculty Member in the Department of Electrical Engineering and Computer Sciences, University of California, Berkeley, where he is currently an Associate Professor. During the 1992-1993 academic year, he was on industrial leave with National Semiconductor, Santa Clara, CA. His research interests include high-frequency power conversion circuits and components, variable-speed ac motor drive systems, simulation, and nonlinear circuit and system theory as related to the power electronics field.

Dr. Sanders has served as Chair of the IEEE Technical Committee on Computers in Power Electronics and was the recipient of the National Science Foundation Young Investigator Award in 1993. 